Exchange bias beyond the superparamagnetic blocking temperature of the antiferromagnet in a $\mathrm{Ni}-\mathrm{NiO}$ nanoparticulate system

Aparna Roy', J. A. De Toro, V. S. Amaral, P. Muniz, J. M. Riveiro, and J. M. F. Ferreira

Citation: Journal of Applied Physics 115, 073904 (2014); doi: 10.1063/1.4866196

View online: http://dx.doi.org/10.1063/1.4866196

View Table of Contents: http://aip.scitation.org/toc/jap/115/7

Published by the American Institute of Physics

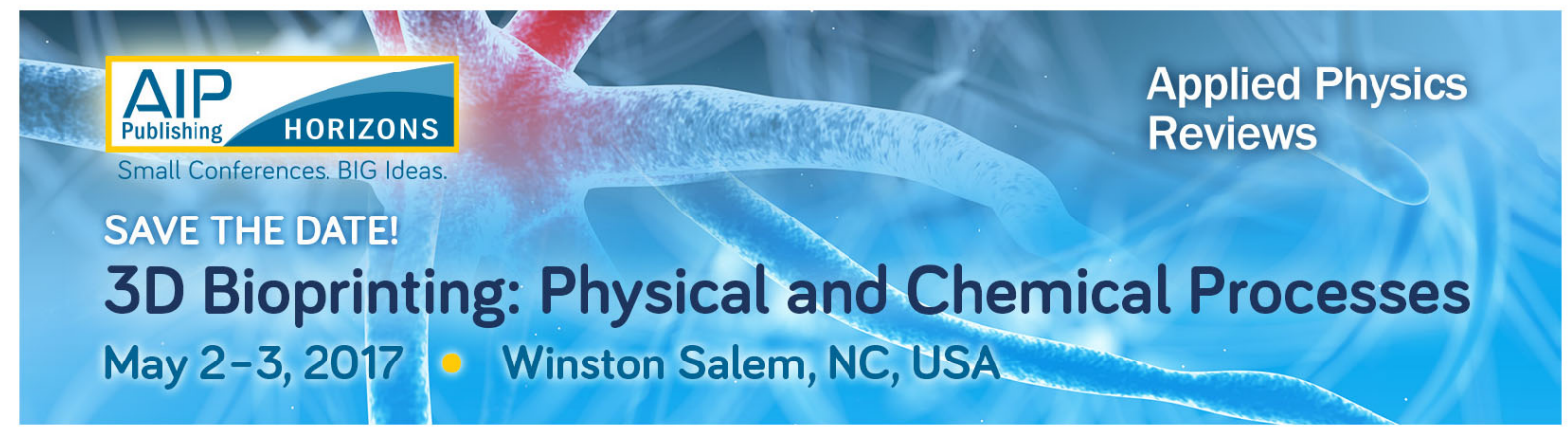




\title{
Exchange bias beyond the superparamagnetic blocking temperature of the antiferromagnet in a Ni-NiO nanoparticulate system
}

\author{
Aparna Roy, ${ }^{1, a)}$ J. A. De Toro, ${ }^{2}$ V. S. Amaral, ${ }^{3}$ P. Muniz, ${ }^{2}$ J. M. Riveiro, ${ }^{2}$ and J. M. F. Ferreira ${ }^{1}$ \\ ${ }^{1}$ Department of Materials and Ceramics Engineering and CICECO, University of Aveiro, 3810193 Aveiro, \\ Portugal \\ ${ }^{2}$ Departamento de Fisica Aplicada, Universidad de Castilla La Mancha, 13071 Ciudad Real, Spain \\ ${ }^{3}$ Department of Physics and CICECO, University of Aveiro, 3810193 Aveiro, Portugal
}

(Received 10 January 2014; accepted 6 February 2014; published online 21 February 2014)

\begin{abstract}
We report magnetic and exchange bias studies on $\mathrm{Ni}-\mathrm{NiO}$ nanoparticulate systems synthesized by a two-step process, namely, chemical reduction of a Ni salt followed by air annealing of the dried precipitate in the temperature range $400550^{\circ} \mathrm{C}$. Size of $\mathrm{Ni}$ and $\mathrm{NiO}$ crystallites as estimated from $X$ ray diffraction line broadening ranges between $10.513 .5 \mathrm{~nm}$ and $2.34 \mathrm{~nm}$, respectively. The magneto-thermal plots (M-T) of these bi-magnetic samples show a well developed peak in the vicinity of $130 \mathrm{~K}$. This has been identified as the superparamagnetic blocking temperature " $\mathrm{T}_{\mathrm{B}}$ " of $\mathrm{NiO}$. Interestingly, all samples exhibit exchange bias even above their respective NiO blocking temperatures, right up to $300 \mathrm{~K}$, the maximum temperature of measurement. This is in contrast to previous reports since exchange bias requires the antiferromagnetic $\mathrm{NiO}$ to have a stable direction of its moment in order to pin the ferromagnet $(\mathrm{Ni})$ magnetization, whereas such stability is unlikely above $\mathrm{T}_{\mathrm{B}}$ since the $\mathrm{NiO}$ is superparamagnetic, its moment flipping under thermal activation. Our observation is elucidated by taking into account the core-shell morphology of the Ni-NiO nanoparticles whereby clustering of some of these nanoparticles connects their $\mathrm{NiO}$ shells to form extended continuous regions of $\mathrm{NiO}$, which because of their large size remain blocked at $\mathrm{T}>\mathrm{T}_{\mathrm{B}}$, with thermally stable spins capable of pinning the $\mathrm{Ni}$ cores and giving rise to exchange bias. The investigated samples may thus be envisaged as being constituted of both isolated core-shell Ni-NiO nanoparticles as well as clustered ones, with $\mathrm{T}_{\mathrm{B}}$ denoting the blocking temperature of the $\mathrm{NiO}$ shell of the isolated particles. (C) 2014 AIP Publishing LLC. [http://dx.doi.org/10.1063/1.4866196]
\end{abstract}

\section{INTRODUCTION}

Exchange bias (EB) is a phenomenon arising due to the exchange coupling created at the interface between a ferromagnet (FM) and an antiferromagnet (AFM) when a system with such interfaces is cooled in a static magnetic field through the Neel temperature $\left(\mathrm{T}_{\mathrm{N}}\right)$ of the AFM. ${ }^{1}$ The phenomenon is typically observed at temperatures below $T_{N}$, though there also exist reports of EB observation at $T>T_{N}$ in some layered systems. ${ }^{2,3}$ Exchange bias has been studied in numerous systems mostly in the form of thin films and occasionally, fine particles. However, past suggestions of EB as a means to delay superparamagnetic (SPM) fluctuations in magnetic nanoparticles ${ }^{4}$ (NPs) and its direct implication in high density magnetic recording have stimulated an upsurge of research on this phenomenon in magnetic nanoparticulate systems.

In order to generate the EB effect, a material must contain two magnetic phases: a reversible phase whose magnetic moment follows the applied magnetic field, and a fixed phase whose moment cannot be reversed in the field range of measurements, i.e., it should be of high magnetic anisotropy. The former can be a FM or ferrimagnet while the latter is customarily an AFM. The AFM should not only remain nonreversible by magnetic field, it should first be thermally stable at the temperature of measurement. Now, in

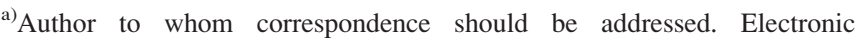
addresses: aparna.roy@ua.pt and aparnaroy15@gmail.com
}

nanoparticulate systems, the AFM due to its small size is likely to be SPM above a certain temperature which implies spontaneous fluctuation of its moment under thermal activation and its inability to pin the FM magnetization. The existence of EB in such systems is thus limited to temperatures below the SPM blocking temperature of the AFM component. ${ }^{5,6}$ Thus, an attempt to combat the SPM limit in nanoparticulate magnetic recording media utilizing the EB effect seems to be thwarted by an additional problem overcoming superparamagnetic fluctuations in the AFM component, more specifically those with small magnetic anisotropy.

Our investigation on exchange bias in Ni-NiO nanoparticulate systems is aimed at addressing this concern and additionally, optimizing the EB effect, if any, at room temperature $(\mathrm{RT}, 300 \mathrm{~K})$. The choice of $\mathrm{Ni}-\mathrm{NiO}$ stems from the fact that the high Neel temperature of $\mathrm{NiO}(523 \mathrm{~K})$ ensures suitability of these systems for EB studies over a wide temperature range, $5523 \mathrm{~K}$, inclusive of room temperature. In this paper, we report the results of this investigation the existence of EB beyond the SPM blocking temperature of $\mathrm{NiO}$, right up to the maximum measurement temperature of $300 \mathrm{~K}$. To our knowledge, this is the first report of its kind for any fine particle system unusual and in contrast to convention. Some such unusual results previously reported in EB systems include the observation of EB on zero field cooling a system from an un-magnetized state ${ }^{7}$ or occurrence of EB when $\mathrm{T}_{\mathrm{C}}<\mathrm{T}_{\mathrm{N}}{ }^{8,9}$ and also when $\mathrm{T}_{\mathrm{C}} \ll \mathrm{T}_{\mathrm{N}},{ }^{10} \mathrm{~T}_{\mathrm{C}}$ being the Curie temperature of the FM component. 


\section{EXPERIMENTAL DETAILS}

Fine particles of $\mathrm{Ni}$ were prepared by chemically reducing the nickel salt $\mathrm{NiCl}_{2} \cdot 6 \mathrm{H}_{2} \mathrm{O}$ (nickel chloride hexahydrate, Sigma Aldrich, Germany) with sodium borohydride $\left(\mathrm{NaBH}_{4}\right.$, Alfa Aesar, Germany) as reducing agent. The reaction was carried out at room temperature. $400 \mathrm{ml}$ of a $1 \mathrm{M}$ (molar) solution of $\mathrm{NaBH}_{4}$ was added dropwise over a period of $2 \mathrm{~h}$ to $2000 \mathrm{ml}$ of a $0.1 \mathrm{M} \mathrm{NiCl}_{2} \cdot 6 \mathrm{H}_{2} \mathrm{O}$ solution in a beaker, under constant magnetic stirring. An instantaneous exothermic reaction ensued with the formation of a black precipitate and evolution of $\mathrm{H}_{2}$ gas. After the completion of reaction, the precipitate was filtered and washed thoroughly with distilled water several times to remove all residual ions from the reaction mixture. It was then left for drying in an oven at $40^{\circ} \mathrm{C}$ for one week. The dried powder is black in colour and stable in ambient atmosphere. It is labelled as "as-prepared" sample. The as-prepared sample was annealed in air at different temperatures in the range $300650^{\circ} \mathrm{C}$ at intervals of $50^{\circ} \mathrm{C}$, for $1 \mathrm{~h}$, to study the evolution of $\mathrm{Ni}$ and $\mathrm{NiO}$ phases.

Crystalline structure and phase evolution of the annealed samples was studied by X-ray diffraction (XRD) using a Rigaku Geigerflex DMax III/C X-ray diffractometer with filtered $\mathrm{Cu} \mathrm{K}$ radiation of wavelength $1.5406 \AA$. The XRD data were recorded in the $2 \theta$ range $25^{\circ} 100^{\circ}$ with a step size of $0.02^{\circ}$. The magneto-thermal behaviour, i.e., the field-cooled (FC)/zero-field-cooled (ZFC) data were recorded in the temperature range $5300 \mathrm{~K}$ with a vibrating sample magnetometer (Cryogenics, UK). The exchange bias measurements were made between 80 and $300 \mathrm{~K}$ in a LDJ Electronics vibrating sample magnetometer with a $1.5 \mathrm{~T}$ electromagnet and fitted with a Hall probe for measuring the field.

We mention here that for clarity of understanding, the annealing temperatures will be expressed in degree centigrade $\left({ }^{\circ} \mathrm{C}\right)$ and the temperatures related to magnetic measurements, in Kelvin $(\mathrm{K})$.

\section{RESULTS AND DISCUSSION}

\section{A. X-ray diffraction and microstructure}

Figure 1 shows the $\mathrm{X}$-ray diffraction profiles of samples annealed in the temperature range $400550^{\circ} \mathrm{C}$. All four samples contain both $\mathrm{Ni}$ and $\mathrm{NiO}$, with $\mathrm{Ni}$ content diminishing and oxide content growing with increasing temperature as indicated by the diminution and enhancement of respective peak intensities. These samples with both metal and metal oxide phase are the ones relevant for EB studies. They are denoted as $S_{400}, S_{450}, S_{500}$, and $S_{550}$, the suffix indicating annealing temperature. Samples annealed at 300, 350, 600, and $650{ }^{\circ} \mathrm{C}$ do not contain both phases and hence are not shown here. A broad hump is seen in the profile of $\mathrm{S}_{400}$ in the $2 \theta$ range $33.6^{\circ} 36.5^{\circ}$. This is related to oxygen-stabilized tetragonal $\mathrm{Ni}(\mathrm{t}-\mathrm{Ni})$, earlier proposed in Ref. 11 as being the end product of the borohydride reduction technique and indexed thereafter and possibly in a remanent state in this sample.

Broad peaks in the diffractograms are indicative of small size of the existing phases. However, since the preparation technique does not allow active control over particle size, it may be envisaged that there is a rather broad distribution of

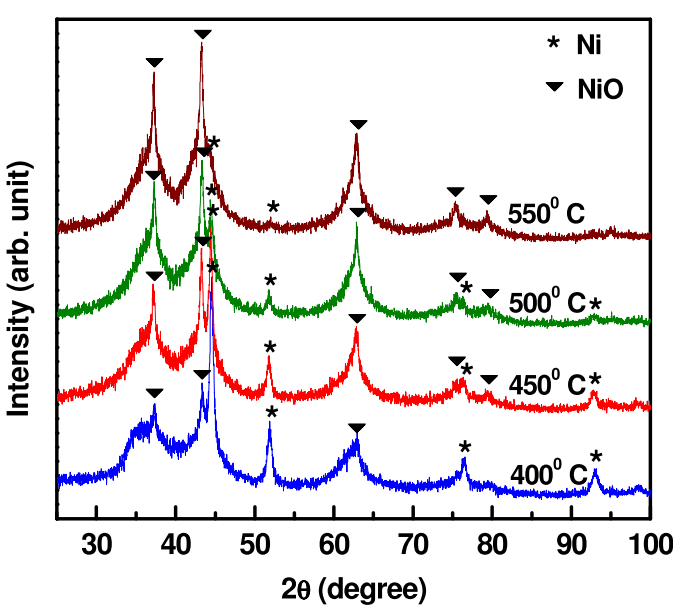

FIG. 1. X ray diffraction profile of the samples with corresponding anneal ing temperature mentioned adjacent to each profile. A small hump is seen in the diffractogram of $\mathrm{S}_{400}$ in the $2 \theta$ range $33.6^{\circ} 36.4^{\circ}$. This is speculated to be due to $\mathrm{t} \mathrm{Ni}$.

particle sizes, from the nanometric single crystallite size to that of polycrystalline particles, though for $\mathrm{NiO}$ we speculate from our reasoning in Sec. III B that a comparatively narrow size distribution prevails.

Figure 2 outlines the TEM micrograph of sample $\mathrm{S}_{450}$, with the inset showing representative core $(\mathrm{Ni})$ shell $(\mathrm{NiO})$ particles of the same. Dark regions against a light background are observed in the main frame. The dark regions signify $\mathrm{Ni}$ while the light background corresponds to $\mathrm{NiO}$, the content of the former being greater than the latter in close conformity with XRD results. The particle size as estimated from the inset micrograph is about $55 \mathrm{~nm}$, with a core size and shell thickness of $40 \mathrm{~nm}$ and $7.5 \mathrm{~nm}$, respectively. From the crystallite size listed in Table I, this hints at a polycrystalline nature of both phases.

$\mathrm{Ni}$ and $\mathrm{NiO}$ crystallite size in the samples as calculated from XRD line broadening using Scherrer equation is

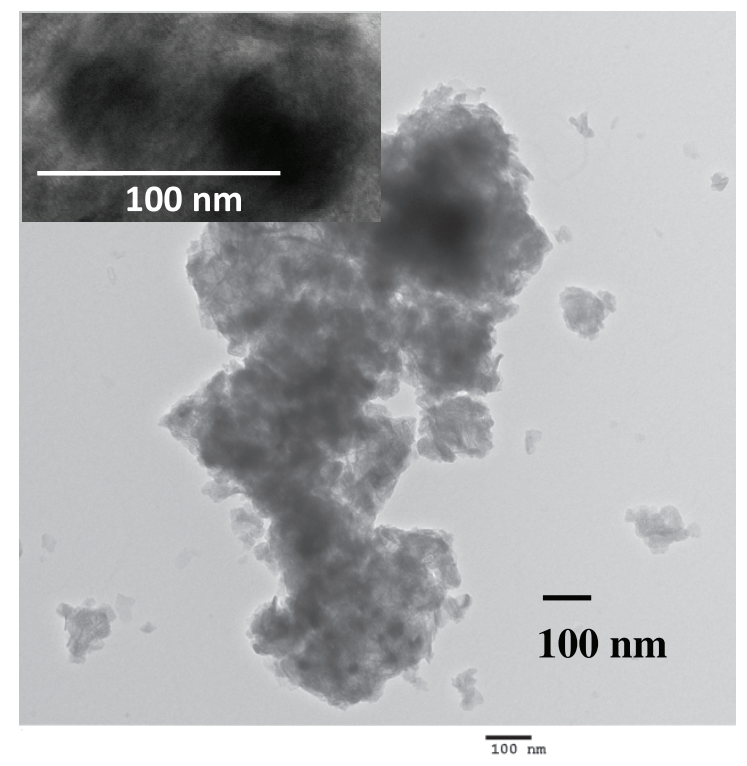

FIG. 2. TEM micrograph of $\mathrm{S}_{450}$. The inset shows core shell particles of the sample. 
TABLE I. Comparison of crystallite size (in "nm") of $\mathrm{Ni}$ and $\mathrm{NiO}$ for differ ent samples.

\begin{tabular}{lrrrr}
\hline \hline Sample & $\mathrm{S}_{400}$ & $\mathrm{~S}_{450}$ & $\mathrm{~S}_{500}$ & $\mathrm{~S}_{550}$ \\
\hline $\mathrm{Ni}$ & 10.51 & 11.65 & 13.52 & \\
$\mathrm{NiO}$ & 2.32 & 3.48 & 3.61 & 4.01 \\
\hline \hline
\end{tabular}

compared in Table I. Since $\mathrm{NiO}$ grows as a shell, the Scherrer values for this phase represents the average thickness of the shell, more precisely the lateral size of the crystallites constituting the shell. Due to the absence of any proper $\mathrm{Ni}$ peak in the profile of $\mathrm{S}_{550}$, crystallite size of $\mathrm{Ni}$ could not be estimated for this sample. Possibly $\mathrm{Ni}$ is in trace amounts here. As expected, $\mathrm{NiO}$ shell crystallites increase in size with increase in air annealing temperature $\left(T_{a n n l}\right)$, though not radically. On the other hand, the small but apparent increase in Ni crystallite size with $\mathrm{T}_{\text {annl }}$ appears contradictory to expected behaviour. This can be explained in terms of two concurrent annealing driven processes: (a) size reduction of the Ni crystallites due to their oxidation, and (b) their tendency to coalesce or aggregate to form bigger crystallites as is usually observed when a powder material is subjected to heat treatment, the latter process dominating the former.

It may be mentioned here that although the Scherrer equation is widely used to estimate the size of nanometric crystallites, it does not include the instrumental and lattice strain broadening effects always present in an XRD pattern. This causes an under estimate of the crystallite size, the actual size being larger than that predicted by the equation.

\section{B. Magnetic properties}

The thermal variation of magnetization of the samples is compared in Figure 3. The data were recorded in accordance with standard zero field cooling and field cooling protocols, under an applied magnetic field of $200 \mathrm{Oe}$ from $5 \mathrm{~K}$ to $300 \mathrm{~K}$ $\left(390 \mathrm{~K}\right.$ for $\left.\mathrm{S}_{400}\right)$. A complete irreversibility in the $\mathrm{FC}$ and

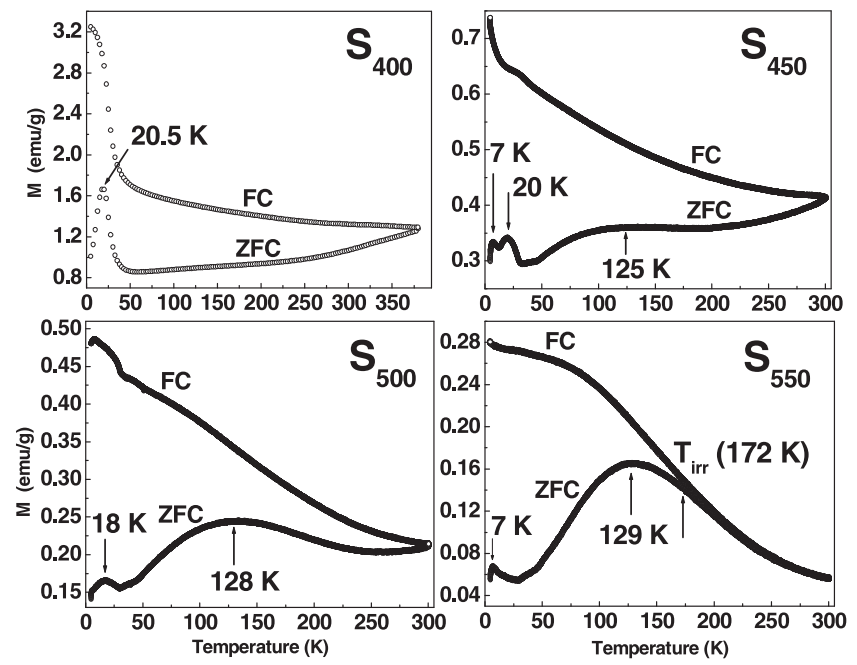

FIG. 3. Magnetization of FC and ZFC particles of the investigated samples as a function of temperature, measured in an applied field of 200 Oe.
ZFC curves is seen right from the starting temperature of $300 \mathrm{~K}$ for $\mathrm{S}_{400}, \mathrm{~S}_{450}$, and $\mathrm{S}_{500}$, indicative of some phase remaining blocked not only at $300 \mathrm{~K}$ but even beyond this temperature. The slope of the ZFC curve at $300 \mathrm{~K}$ allows us to infer this. We attribute this $\mathrm{FC} / \mathrm{ZFC}$ irreversibility to the blocking of the Ni cores of these bi-phase samples. An estimate of the critical size for $\mathrm{Ni}$ nanoparticles to remain blocked, i.e., ferromagnetic at RT, can be obtained from the equation $\mathrm{K}_{\mathrm{Ni}} \times \mathrm{V}=25 \mathrm{~K}_{\mathrm{B}} \mathrm{T}_{\mathrm{B}}$ [V the particle volume; $\mathrm{K}_{\mathrm{B}}$ the Boltzmann Constant; $\mathrm{K}_{\mathrm{Ni}}=5 \times 10^{4} \mathrm{erg} / \mathrm{cm}^{3}$ is the anisotropy of $\mathrm{Ni}$; $\mathrm{T}_{\mathrm{B}}$ the blocking temperature, $300 \mathrm{~K}$ ] which shows that they should be larger than about $34 \mathrm{~nm}^{.2}$ While the relatively large Ni cores of the size distribution with each core comprising several small crystallites strongly coupled by direct exchange conform to this critical value and hence are blocked at $\mathrm{T} \geq 300 \mathrm{~K}$, the crystallites with size ranged between 10.5 and $13.5 \mathrm{~nm}$ and other small $\mathrm{Ni}$ cores of the distribution fall far short of the critical value, thereby raising an open question on their RT stability. This apparent anomaly can be accounted by the different anisotropies of the cores, including the uniaxial anisotropy introduced by the coupling or interaction between spins at the FM (Ni) AFM (NiO) interface. ${ }^{12}$ The net result is an enhancement in the anisotropy of these cores which concomitantly raises the energy barrier to moment reversal, leading to their thermal stability or blocked state at $300 \mathrm{~K}$.

A broad peak at $125 \mathrm{~K}$ is observed in the ZFC curve of $\mathrm{S}_{450}$, progressively sharpening to 128 and $129 \mathrm{~K}$ in samples $\mathrm{S}_{500}$ and $\mathrm{S}_{550}$ respectively, and pointing to the blocking of some phase at these temperatures. The blocked state of the $\mathrm{Ni}$ cores at $300 \mathrm{~K}$ precludes them from being related to these peaks. We therefore propose that these peaks represent the average SPM blocking temperature " $\mathrm{T}_{\mathrm{B}}$ " of the $\mathrm{NiO}$ component in the respective samples. The fact that these peaks sharpen and become prominent with $\mathrm{T}_{\mathrm{annl}}$ lends credence to our proposition. Sample $\mathrm{S}_{400}$ does not show this peak probably because of low $\mathrm{NiO}$ content. It is also observed that $\mathrm{S}_{550}$ shows $\mathrm{FC} / \mathrm{ZFC}$ irreversibility from $172 \mathrm{~K}$, in contrast to $300 \mathrm{~K}$ of other samples. This temperature $(172 \mathrm{~K})$ designated as $\mathrm{T}_{\text {irr }}$ signifies the blocking corresponding to the largest $\mathrm{NiO}$ particles $(7.2 \mathrm{~nm}$ as calculated from the equation $\left.\mathrm{K}_{\mathrm{NiO}} \times \mathrm{V}=25 \mathrm{~K}_{\mathrm{B}} \mathrm{T}_{\mathrm{B}}\left[\mathrm{K}_{\mathrm{NiO}}=2.8 \times 10^{6} \mathrm{erg} / \mathrm{cm}^{3}\right]\right)$ in the size distribution. Since $S_{550}$ is the most oxidized sample of the investigated series, its largest $\mathrm{NiO}$ size may be considered as an upper limit to the $\mathrm{NiO}$ size in the remaining less oxidized samples, $S_{450}$ and $S_{500}$. We thus speculate the thickness of the $\mathrm{NiO}$ shell to be in the range $3.57 .2 \mathrm{~nm}$, with a polycrystalline nature of the shell constituents.

Our proposition of the ZFC peaks near $130 \mathrm{~K}$ being representative of $\mathrm{NiO}$ is further ratified quantitatively when on subtracting the background FM signal [approximately $\left.\left(\mathrm{M}_{300 \mathrm{~K}}+\mathrm{M}_{5 \mathrm{~K}}\right) / 2\right]$ on which the peaks are superposed, from the overall signal $(0.36,0.25,0.165 \mathrm{emu} / \mathrm{g})$ to estimate the absolute size of the peaks, we obtain an increasing size from 0.005 to 0.072 and finally $0.108 \mathrm{emu} / \mathrm{g}$ for $\mathrm{S}_{450}, \mathrm{~S}_{500}$, and $\mathrm{S}_{550}$ respectively. Thus, despite the overall loss of magnetization due to the transformation of $\mathrm{Ni}$ to $\mathrm{NiO}$, the absolute signal associated with these peaks increases in close conformity with the increasing $\mathrm{NiO}$ content of these samples. It is 
important to note here that $a Z F C$ peak ascribed to the blocking of the AFM component in a study of exchange bias in FM AFM coupled systems is rather rare. The peaks customarily observed are due to the blocking of the FM cores, the AFM contribution being typically masked by the FM signal. In contrast, the intriguing feature of our samples is that the AFM contribution is well visible in the magnetization study, both in the M-T plots and in the linear non-saturating component of M-H loops (Figure 4).

As regards the low temperature features, a peak at $\sim 20 \mathrm{~K}$ is observed in the ZFC curve of samples $\mathrm{S}_{400}, \mathrm{~S}_{450}$, and $S_{500}$, though in the latter two it is very much diminished. This peak is related to a Curie-like Paramagnet to FM transition of the randomly oriented spins of oxygen-stabilized tetragonal Ni, vide detailed explanation in Ref. 13. In this context, we mention that although $\mathrm{t}-\mathrm{Ni}$ is expected to get almost completely converted to fcc $\mathrm{Ni}$ on heat treating the as-prepared sample at and above $400^{\circ} \mathrm{C}$, some traces might still be left in samples $S_{450}$ and $S_{500}$, not clearly discernible by XRD but leaving its finger print on the magneto-thermal behaviour. In addition to the peak at $20 \mathrm{~K}$, another low temperature peak is seen at $7 \mathrm{~K}$ in the ZFC curve of samples $S_{450}$ and $S_{550}$ but non-existent in $S_{500}$. We believe this to be an artefact of the instrument as it is not consistent in its appearance.

\section{Exchange bias studies}

\section{Shifted M-H loops}

Figure 4 displays the field variation of magnetization (M-H loops) at $160 \mathrm{~K}$, recorded after cooling the samples in a field of $11 \mathrm{kOe}$ from $490 \mathrm{~K}$ to the measuring temperature.
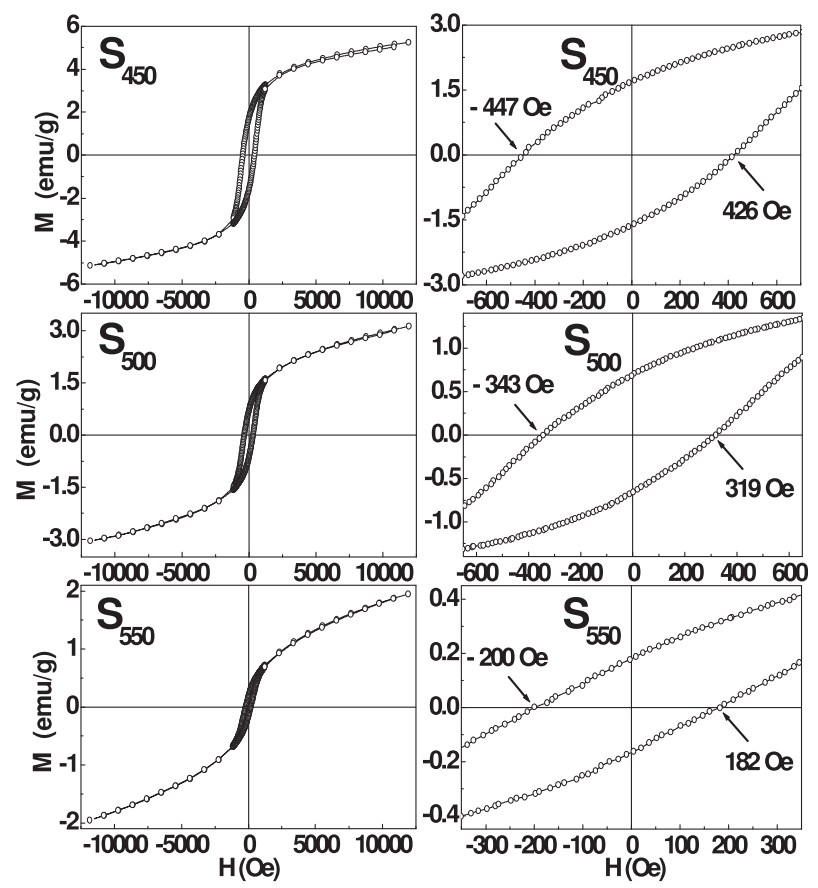

FIG. 4. Hysteresis loops at $160 \mathrm{~K}$, recorded after cooling the samples from $490 \mathrm{~K}$ to the measuring temperature in a field of $11 \mathrm{kOe}$. Left panel shows the complete loop while the right panel depicts an expanded view. Coercivities of the left and right branch of the loops are marked on each figure.
The complete loop is shown in the left panel while an expanded view of each loop is highlighted in the right one. The loops are shifted from the zero field position to the left and hence asymmetric with respect to field axis, confirming the existence of EB. It is also evident that the M-H loops of $\mathrm{S}_{450}$ and $\mathrm{S}_{500}$ are closed at $\mathrm{H} \sim 2.3 \mathrm{kOe}$, implying saturation of the FM magnetization and hence allowing us to conclude that the observed shifts are not due to minor loops ${ }^{1}$ hysteresis loops which also exhibit different coercivities for the increasing and decreasing field branches when the FM component is unsaturated. As for sample $\mathrm{S}_{550}$ which is almost completely oxidized, EB appears due to a reason different from $\mathrm{S}_{450}$ and $\mathrm{S}_{500}$ (Sec. III C 2).

The EB field, $H_{\mathrm{E}}$, defined by the formula $H_{E}=\frac{\left|H_{C}^{+}\right|\left|H_{C}\right|}{2}$ is a measure of the shift of the hysteresis loop along the field axis. The calculated values of this shift at $160 \mathrm{~K}$ are 10.5 , 11.5 , and 9 Oe for samples $S_{450}, S_{500}$, and $S_{550}$ respectively. The small magnitude of $\mathrm{H}_{\mathrm{E}}$ is usually attributed to the small anisotropy of $\mathrm{NiO}$, though the density of FM AFM interface area is an equally important factor for obtaining significant bias. Existence of large particles in a size distribution implies reduction of this interface density and hence of the EB field, $\mathrm{H}_{\mathrm{E}}$. Likewise, the small magnitude of $\mathrm{H}_{\mathrm{E}}$ above the $\mathrm{NiO}$ blocking temperature " $\mathrm{T}_{\mathrm{B}}$ " may be accounted by the smallness in the number of thermally stable "large AFM regions" (Sec. III C) a must for EB above $\mathrm{T}_{\mathrm{B}}$ in addition to the two above mentioned reasons. We have not measured EB for $\mathrm{S}_{400}$ since it does not show any perceptible peak in the ZFC curve that can be assigned to NiO. This, however, does not imply the absence of $\mathrm{NiO}$ in the sample but rather its low content.

The temperature variation of the EB field, $\mathrm{H}_{\mathrm{E}}$, is shown in Figure 5. The inset shows the error bars in $\mathrm{H}_{\mathrm{E}}$ for one of the samples, $\mathrm{S}_{450}$. It is clear that for all samples we can still have non-zero bias at $\mathrm{T}>300 \mathrm{~K}$. As expected, EB decreases with increasing temperature due to the reduction in the magnetocrystalline anisotropy of NiO. Figure 5 also evidences a decrease in $\mathrm{H}_{\mathrm{E}}$ with increase in $\mathrm{T}_{\mathrm{ann}}$, though there is a crossover in the $\mathrm{H}_{\mathrm{E}}$ values of samples $\mathrm{S}_{450}$ and $\mathrm{S}_{500}$ at $\mathrm{T} \geq 160 \mathrm{~K}$. The reduction in $\mathrm{H}_{\mathrm{E}}$ with $\mathrm{T}_{\mathrm{annl}}$ can be understood as being governed by the improvement in crystallinity of the FM AFM interface when subject to thermal treatment. This smears out the interface roughness, reducing in the process the number of uncompensated spins at the interface that are responsible for exchange bias. We are, however, yet to comprehend the reverse trend in the exchange bias values of $S_{450}$ and $S_{500}\left(H_{E}\right.$ increasing with $\left.T_{\text {annl }}\right)$ at $T \geq 160 \mathrm{~K}$. Considering the closeness of the $\mathrm{H}_{\mathrm{E}}$ values, an overlap of their regions of error is quite plausible. It would not then be unreasonable to envisage the $\mathrm{H}_{\mathrm{E}}$ values as following the expected trend at $\mathrm{T} \geq 160 \mathrm{~K}$ as well.

Although of small magnitude, EB exists in all samples at all temperatures over the complete range of measurement $80300 \mathrm{~K}$, including as is seen, temperatures above the SPM blocking temperature of $\mathrm{NiO}$. We mention here that due to instrumental limitations the field cooling of samples was done from $490 \mathrm{~K}$ and not from above $523 \mathrm{~K}$, the Neel temperature of $\mathrm{NiO}$. Enhanced values of $\mathrm{H}_{\mathrm{E}}$ might be obtained in case of field cooling from above $523 \mathrm{~K}$. 


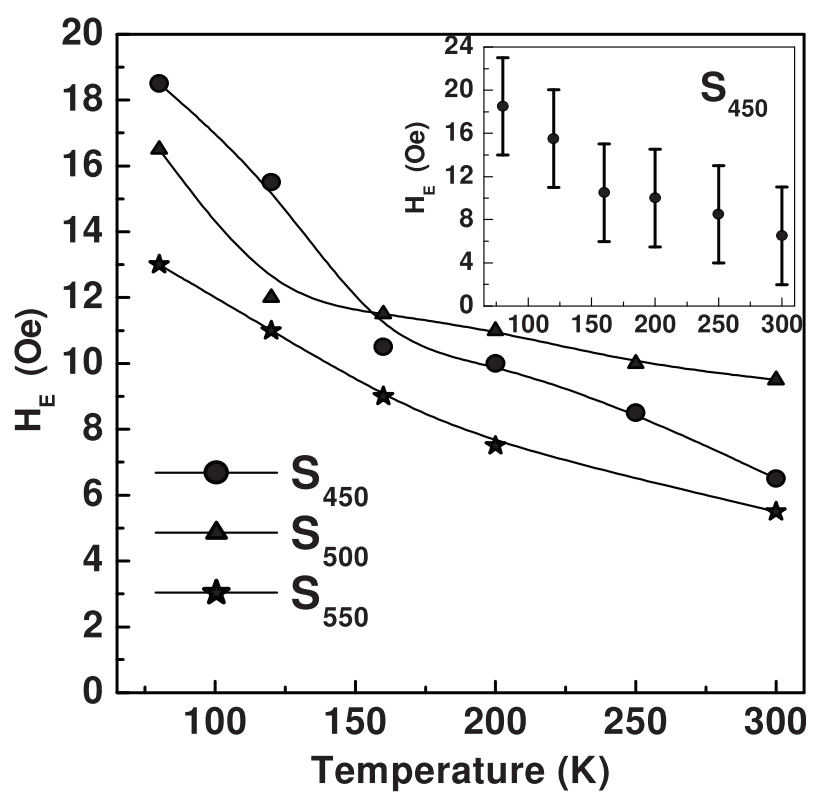

FIG. 5. Temperature variation of the exchange bias field $\mathrm{H}_{\mathrm{E}}$. The lines are guides to the eye. Inset shows the error bars in $\mathrm{H}_{\mathrm{E}}$ for sample $\mathrm{S}_{450}$.

\section{Exchange bias above $T_{B}$ :Analysis}

Figure 6(a) portrays a pictorial representation of an isolated core-shell $\mathrm{Ni}-\mathrm{NiO}$ nanoparticle a $\mathrm{Ni}$ core of size $12 \mathrm{~nm}$ surrounded by a $\mathrm{NiO}$ shell of thickness $3.5 \mathrm{~nm}$. The sizes mentioned correspond to the Scherrer size of both phases. However, such a core-shell picture is applicable to all particles of the size distribution with an obvious variation in core size and shell thickness. The narrow thickness of the $\mathrm{NiO}$ shell implies symmetry breaking at its surface, resulting in uncompensated spins and subsequent SPM behaviour above a blocking temperature $T_{B}$. It is imperative that to generate the EB effect in a FM AFM bi-magnetic system at a particular temperature, the first pre-requisite is the thermal stability of the AFM at that temperature. A superparamagnetic $\mathrm{NiO}$ in an isolated core-shell $\mathrm{Ni}-\mathrm{NiO}$ nanoparticle cannot, with its thermally fluctuating spins, pin the $\mathrm{Ni}$ spins at the interface, thereby nullifying the possibility of EB at temperatures above $\mathrm{T}_{\mathrm{B}}$.

However, if several such core-shell NPs come into contact, their AFM shells join to form a large extended region of
NiO. This is illustrated in Figure 6(b). Such extended, continuous regions can maintain thermally stable AFM spin orientations over their complete expanse even above $T_{B}$ since their large size prohibits spin reversal. Now, given the fact that core-shell NPs naturally incorporate roughness at the interface vital for giving rise to uncompensated spins it is logical to expect the $\mathrm{NiO}$ sublattice magnetization to be uncompensated at the interface between $\mathrm{Ni}$ and $\mathrm{NiO}$. It is these uncompensated spins, anchored in the AFM or at the FM/AFM interface ${ }^{14}$ that exchange couple to the interface FM spins, giving rise to $\mathrm{EB}$ at temperatures higher than $T_{B}$, now that the AFM spin stability is ensured at such temperatures by large extended $\mathrm{NiO}$ regions which may justifiably be assumed to remain blocked at $\mathrm{T}>\mathrm{T}_{\mathrm{B}}$, right up to RT and even above. Samples $\mathrm{S}_{450}$ and $\mathrm{S}_{500}$ may thus be envisaged as being constituted of both isolated core-shell $\mathrm{Ni}$-NiO nanoparticles as well as clustered ones, with the bias below $\mathrm{T}_{\mathrm{B}}$ contributed by both sets of NPs while that above $\mathrm{T}_{\mathrm{B}}$ accounted by only the clustered ones, small in number and hence a minority. $T_{B}$ denotes the SPM blocking temperature of the NiO shell of the isolated particles.

As regards the exchange bias exhibited by $\mathrm{S}_{550}$, we recall that it is almost completely oxidized to $\mathrm{NiO}, \mathrm{Ni}$ being in trace amounts. In such a scenario $S_{550}$ may be considered, without much error, to be mostly composed of single phase AFM nanoparticles. Exchange bias in such single phase AFM nanoparticles can be ascribed to the exchange coupling between an AFM core and a FM or spin-glass-like (SGL) or SPM shell originating from uncompensated surface spins. ${ }^{15}$ The AFM core plays the role of fixed phase while the uncompensated surface spins constituting the shell play the role of reversible phase (FM), much alike a conventional FM-AFM exchange biased system. Although the AFM core remains stable at temperatures both above and below $T_{B}$ $(129 \mathrm{~K})$, the uncompensated surface spins fluctuate thermally above this temperature. In order to contribute to $\mathrm{EB}$, they should first be thermally stable so that magnetic field remains the only parameter to control their reversal. Clustering of the single phase NiO NPs connects their SGL or SPM shells, making the surface region housing these spins a big extended one. This translates to an enhanced energy barrier to moment reversal, resulting in thermal stability of the spins even above $\mathrm{T}_{\mathrm{B}}$ and subsequent display of $\mathrm{EB}$ by the sample.

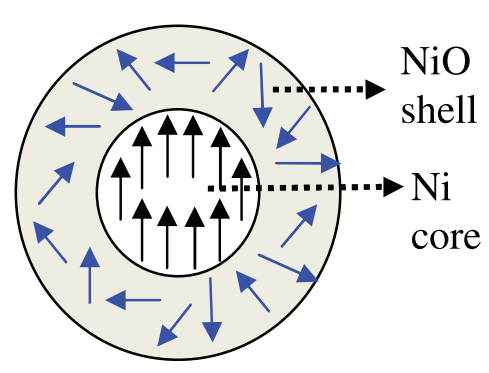

(a)

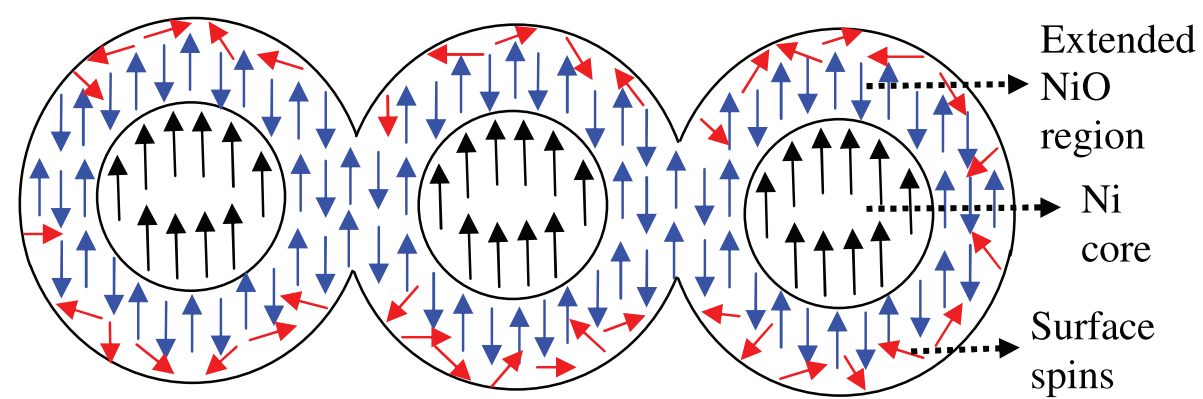

(b)

FIG. 6. (a) Speculated spin configuration in a core shell structured Ni NiO nanoparticle. Clustering of several such NPs leads to an extended, continuous region of $\mathrm{NiO}$ with the $\mathrm{Ni}$ cores appearing dispersed in it. (b) Spin orientations over the extended region and at its interface with the cores. This is a simplified picture, conjectured and illustrated for the ease of understanding. The actual spin configuration may be more complex. 
In the context of our analysis of exchange bias above $T_{B}$ based on the proposed shell-shell interaction model, we mention that similar models have earlier been proposed in literature $^{16,17}$ to elucidate the radically enhanced magnetic stability of FM cores of core-shell FM-AFM nanoparticles in contact, as compared to when the NPs were isolated. The enhanced shell thickness when the NPs come into contact was proposed to recover the bulk AFM properties of the shell, leading to better interfacial coupling, large bias fields, and an increase in the blocking temperature of the cores. In the present analysis, however, connectivity of the $\mathrm{NiO}$ shells and the resulting extended $\mathrm{NiO}$ regions have been invoked to account for the magnetic stability (thermally stable spin orientations) of the very shell itself, the enhanced energy barrier due to extended shell size prohibiting spin reversals in it.

Finally, it is relevant to analyze here the feasibility of the "magnetic proximity effect" as being another origin of the thermally stable AFM ordering above the AFM's superparamagnetic blocking temperature. The effect has been observed in both thin film ${ }^{18}$ and nanoparticulate systems. ${ }^{19}$ Theoretical calculations of FM/AFM thin film bilayers indicate that the subsystem with the larger ordering temperature induces magnetic order into the other one by providing a local magnetic field. Furthermore for FM/AFM bilayers with $\mathrm{T}_{\mathrm{C}}>\mathrm{T}_{\mathrm{N}}$, neutron diffraction studies have established that the Neel temperature of the AFM can be enhanced by its proximity to the FM. Considering these facts, it may be reasoned that since the Ni cores of the presently investigated samples are ferromagnetic (blocked), i.e., ordered above RT, they may induce AFM order into the $\mathrm{NiO}$ shells by virtue of their proximity. However, due to small shell thickness the AFM spin configuration will flip coherently at temperatures above $\mathrm{T}_{\mathrm{B}}$ from one easy direction to the other, maintaining the AFM order all along. Hence, although a "proximity effect" induced AFM order may exist in the shells, it (order) is not thermally stable above $T_{B}$. Thermal stability of AFM spin orientations above $T_{B}$ can ensue in the event of the shells joining together and forming extended $\mathrm{NiO}$ regions up on clustering of the NPs, as explained in the text.

\section{CONCLUSIONS}

In summary, we have studied the exchange bias effect in $\mathrm{Ni}-\mathrm{NiO}$ nanoparticulate systems prepared by the borohydride reduction of a $\mathrm{Ni}$ salt followed by air annealing of the dried precipitate. The maximum RT exchange bias (10 Oe) is exhibited by sample $S_{500}$. Exchange bias decreases with increasing annealing temperature due to the crystallinity improvement at the $\mathrm{Ni}-\mathrm{NiO}$ interface. All samples show EB even at temperatures above the SPM blocking temperature of $\mathrm{NiO}$, in contrast to previous reports. This is explained by considering that clustering of some of these core-shell NPs forms extended continuous regions of $\mathrm{NiO}$ which being large, remain blocked at $\mathrm{T}>\mathrm{T}_{\mathrm{B}}$, with thermally stable spins capable of pinning the $\mathrm{Ni}$ particles and giving rise to $\mathrm{EB}$. Although much research needs to be done to make these systems amenable to technological applications, we are tempted to think of their use as magnetic recording media since superparamagnetism of both the nanosized core and shell can be overcome. The ideal situation would be that with an AFM blocking temperature of $300 \mathrm{~K}$ which may be possible if all (not some) the core-shell NPs get clustered, i.e., there are no isolated NPs. The cores would, however, still remain protected against coalescence by their native oxide shell (now matrix after complete clustering), maintaining their suitability as a viable recording medium.

\section{ACKNOWLEDGMENTS}

A. Roy acknowledges the Foundation of Science and Technology (FCT) of Portugal for financial support under the Grant SFRH/BPD/77574/2011. Support from CICECO, University of Aveiro is gratefully acknowledged.

${ }^{1}$ J. Nogues, J. Sort, V. Langlais, V. Skumryev, S. Surinach, J. S. Munoz, and M. D. Baro, Phys. Rep. 422, 65 (2005).

${ }^{2}$ M. Grimsditch, A. Hoffmann, P. Vavassori, H. Shi, and D. Lederman, Phys. Rev. Lett. 90, 257201 (2003).

${ }^{3}$ X. Chen, Ch. Binek, A. Hochstrat, and W. Kleemann, Phys. Rev. B 65, 012415 (2001).

${ }^{4}$ V. Skumryev, S. Stoyanov, Y. Zhang, G. Hadjipanayis, D. Givord, and J. Nogues, Nature (London) 423, 850 (2003).

${ }^{5}$ S. Gangopadhyay, G. C. Hadjipanayis, C. M. Sorensen, and K. J. Klabunde, J. Appl. Phys. 73, 6964 (1993).

${ }^{6}$ S. K. Sharma, J. M. Vargas, M. Knobel, K. R. Pirota, C. T. Meneses, S. Kumar, C. G. Lee, P. G. Pagliuso, and C. Rettori, J. Appl. Phys. 107, 09D725 (2010).

${ }^{7}$ B. M. Wang, Y. Liu, P. Ren, B. Xia, K. B. Ruan, J. B. Yi, J. Ding, X. G. Li, and L. Wang, Phys. Rev. Lett. 106, 077203 (2011).

${ }^{8}$ X. W. Wu and C. L. Chien, Phys. Rev. Lett. 81, 2795 (1998).

${ }^{9}$ A. Lopez Ortega et al., J. Am. Chem. Soc. 132, 9398 (2010).

${ }^{10}$ J. W. Cai, K. Liu, and C. L. Chien, Phys. Rev. B 60, 72 (1999).

${ }^{11}$ A. Roy, V. Srinivas, S. Ram, J. A. De Toro, and U. Mizutani, Phys. Rev. B 71, 184443 (2005).

${ }^{12}$ J. Nogues, V. Langlais, J. Sort, S. Doppiu, S. Surinach, and M. D. Baro, J. Nanosci. Nanotechnol. 8, 1775 (2008).

${ }^{13}$ A. Roy, V. Srinivas, J. A. De Toro, and J. P. Goff, Phys. Rev. B 74, 104402 (2006).

${ }^{14}$ H. Ohldag, A. Scholl, F. Nolting, E. Arenholz, S. Maat, A. T. Young, M. Carey, and J. Stohr, Phys. Rev. Lett. 91, 017203 (2003). The uncompen sated spins are tightly locked or anchored or pinned to the AFM lattice and hence do not rotate in an external magnetic field.

${ }^{15}$ S. A. Makhlouf, J. Magn. Magn. Mater. 272-276, 1530 (2004).

${ }^{16}$ J. Nogues, V. Skumryev, J. Sort, S. Stoyanov, and D. Givord, Phys. Rev. Lett. 97, 157203 (2006).

${ }^{17}$ G. Margaris, K. N. Trohidou, and J. Nogues, Adv. Mater. 24, 4331 (2012).

${ }^{18}$ J. van Lierop, K. W. Lin, J. Y. Guo, H. Ouyang, and B. W. Southern, Phys. Rev. B 75, 134409 (2007).

${ }^{19}$ I. V. Golosovsky, G. Salazar Alvarez, A. Lopez Ortega, M. A. Gonzalez, J. Sort, M. Estrader, S. Surinach, M. D. Baro, and J. Nogues, Phys. Rev. Lett. 102, 247201 (2009). 